\title{
Kindesschutz: Prävention von Anfang an
}

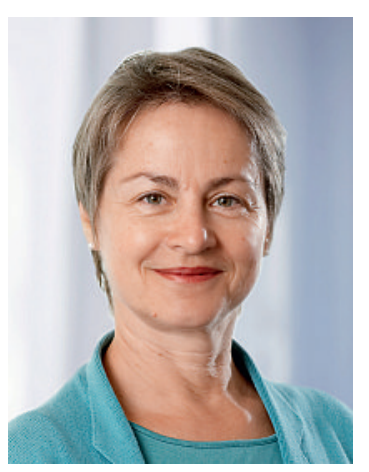

Wieder einmal sitzt mir in meiner psychiatrisch-psychotherapeutischen Praxis eine Patientin gegenüber, die mit kargen Sätzen, unterbrochen von langem Schweigen, von den Misshandlungen erzählt, denen sie als Kind ausgesetzt war. Sie spricht von der Kindergärtnerin, die wohl nachgefragt hat, als sie das blaugeschlagene Auge des Kindes sah, sich aber von der Mutter abspeisen liess mit einer fadenscheinigen Erklärung. Sie spricht vom Kinderarzt, der die Spuren der Schläge mehr als einmal gesehen haben muss, aber nie etwas unternommen hat. Mit leiser Stimme erzählt sie, wie wertlos sie sich als Kind gefühlt habe; niemand hat sich für sie eingesetzt. Ich höre aus den Worten der Patientin aber auch heraus, wie hilflos sich wohl die Kindergärtnerin fühlte. Was es den Kinderarzt gekostet haben mag, seine Arbeit einfach so gut wie möglich zu tun, lässt sich allenfalls erahnen. Ein brutaler Suizidversuch der Mutter zeugt schliesslich auch von deren massiver Überforderung.

Diese Geschichte ist vor mehr als dreissig Jahren geschehen - wir wissen aber, dass Ähnliches sich noch immer in viel zu vielen Familien abspielt. Doch die öffentliche Wahrnehmung von Gewalt gegen Kinder hat sich geändert - der Wille ist da, Kinder zu schützen und Familien zu unterstützen.

\section{Ärztinnen und Ärzte können die Gefährdung eines Kindes frühzeitig erkennen.}

In dieser Ausgabe der Schweizerischen Ärztezeitung werden wichtige Meilensteine auf diesem Weg vorgestellt: Heute kann die Kindergärtnerin sich Rat holen, und der Arzt hat möglicherweise die neue Broschüre «Kindsmisshandlung - Kindesschutz: Ein Leitfaden zu Früherfassung und Vorgehen in der ärztlichen Praxis» vor sich. Verfasst wurde der Leitfaden von der Kinderschutzgruppe und Opferberatungsstelle der Universitäts-Kinderkliniken Zürich unter der Leitung von Dr. med. Ulrich Lips. Die Beratungsstelle berichtet in diesem Heft auf Seite 400 von ihrer Arbeit: Die Zahlen über Kindsmisshandlungen sind zunächst erschreckend; sie zeigen aber auch, dass eine deutliche Sensibilisierung stattgefunden hat. Immer häufiger wenden sich Bezugspersonen an eine Beratungsstelle, wenn sie eine Misshandlung vermuten. Eine wichtige Rolle kommt der kinderärztlichen Vorsorgeuntersuchung $\mathrm{zu}$, sie ist eine der wichtigsten regelmässigen Kontakte ausserhalb der Familie überhaupt. Ärztinnen und Ärzte können die Gefährdung eines Kindes frühzeitig erkennen, der Leitfaden hilft ihnen dabei, indem er Hinweise enthält, ab wann eine Gefährdung besteht, welche Warnsignale es gibt, wie man dem betroffenen Kind helfen kann und wie die Eltern angesprochen werden sollen.

\section{Der Leitfaden der Stiftung Kinder- schutz Schweiz gibt wichtige Hinweise, was Ärzte bei einem Verdacht auf Misshandlungen tun können.}

Der Leitfaden ist von der nationalen Stiftung Kinderschutz Schweiz in Auftrag gegeben worden. Diese Stiftung setzt sich seit Jahren erfolgreich dafür ein, dass alle Kinder in unserer Gesellschaft in Würde und ohne Verletzung ihrer Integrität aufwachsen. Neben Projekten wie beispielsweise der Kinderparcours «Mein Körper gehört mir» als Präventionsarbeit gegen sexuelle Gewalt macht die Stiftung gezielte politische Lobbyarbeit, Beratung und Öffentlichkeitsarbeit zum Thema Kindesschutz. Sie engagiert sich auch aktiv im internationalen Kampf gegen Kinderprostitution, Kinderpornographie und Kinderhandel.

Die FMH unterstützt die Bestrebungen der Stiftung Kinderschutz Schweiz: Kinder müssen vor Gefährdung, Vernachlässigung, Ausbeutung und vor jeglicher Gewalt geschützt werden. Als Ärztinnen und Ärzte können wir dabei einen zentralen Beitrag leisten: Wir empfehlen den vorliegenden Leitfaden als wichtiges Instrument im Berufsalltag, um gefährdeten Kindern frühzeitig Hilfe und Schutz zukommen zu lassen und den betroffenen Müttern und Vätern Unterstützung anbieten zu können. Damit möglichst viele Kinder - so wie es ihnen zusteht - unbeschwert und ohne schweren Rucksack aufwachsen können.

Dr. med. Christine Romann, Mitglied des Zentralvorstands der FMH, Verantwortliche Ressort Gesundheitsförderung und Prävention 